\title{
Pesquisa Convergente Assistencial: uso na elaboração de modelos de cuidado de enfermagem
}

\author{
Convergent Care Research: use in developing models of nursing care
}

Investigación Convergente Asistencial: uso en el desarrollo de modelos de atención de enfermería

Submissão: 03-08-2010 Aprovação: 13-11-2012

\section{RESUMO}

O presente texto é uma reflexão teórica da importância dos modelos de cuidado de Enfermagem para a consolidação dessa disciplina e acerca da contribuição da Pesquisa Convergente Assistencial (PCA) na elaboração de tais modelos. Além disso, discute sua importância na convergência dos meios acadêmico e prático, que auxiliam em uma prática segura, sistematizam o cuidado e estabelecem ligação entre o pragmatismo e o cientificismo, levando os enfermeiros a adotarem posturas de construção de conhecimento mais sólidas.

Descritores: Enfermagem; Cuidado; Metodologia.

\section{ABSTRACT}

The present text is a theoretical reflection upon the importance of Nursing care models for the consolidation of this discipline, concerning the contribution of Convergent Care Research (Pesquisa Convergente Assistencial -PCA) in elaborating such models. Beyond this, this article discusses the importance of Convergent Care Research in the convergence of the academic and practical worlds of nursing, which aid towards a safe practice, systematize care, and establish connections between pragmatism and scientism, leading nurses to adopt more solid knowledge construction postures.

Key words: Nursing; Care; Methodology.

\section{RESUMEN}

En el presente artículo se desarrolla una reflexión teórica sobre la importancia de los modelos de cuidado de enfermería para la consolidación de esa disciplina, y sobre la contribución de la Investigación Convergente Asistencial (ICA) para la elaboración de esos modelos. Además, se analiza su importancia en la convergencia entre el medio académico y el práctico, que ayudan para la realización de una práctica segura, así como para la sistematización del cuidado, sirviendo de enlace entre el pragmatismo y el cientificismo, lo que permite que los enfermeros adopten posturas de construcción del conocimiento más sólidas.

Palabras clave: Enfermería; Cuidado; Metodología.

\section{AUTOR CORRESPONDENTEＰatrícia Kuerten RochaＥ-mail: pkrochaucip@yahoo.com.br}




\section{CONSTRUÇÃO DO CONHECIMENTO EM ENFERMA- GEM: REQUERIMENTO PARA A CONSOLIDAÇÃO DIS- CIPLINAR}

A história da humanidade demonstra que o cuidar sempre esteve presente nas diferentes dimensões do processo de viver, adoecer e morrer, mesmo antes do surgimento das profissões ${ }^{(1)}$. O conceito de cuidar é extremamente discutido e varia conforme o autor, pois há diversas formas de se olhar o cuidador, o ser cuidado e o entorno ${ }^{(2)}$.

O cuidado pode ser realizado de diferentes formas e por profissionais ou não. Quando realizado por profissionais, pode ser visto como um processo que emerge do encontro de dois (ou mais) seres humanos em que cada um detém elementos do processo de cuidado ${ }^{(3)}$. Sendo assim, o cuidado, quando profissional, pode/deve ser compreendido como produto e serviço, nas suas múltiplas dimensões, relações e interações; ou seja, é um sistema de produção de serviços personificado e singular na sua maneira de ser e existir na sociedade ${ }^{(4)}$.

O cuidado prestado por enfermeiros foi testado e comprovado cientificamente no final do século passado, contribuindo para que a disciplina de enfermagem passasse a ser vista como uma ciência, e o cuidado, como sua essência ${ }^{(5)}$.

Percebemos, assim, que o cuidado não se esgota na prática, nem mesmo na pesquisa, estando sempre em processo de renovação. Ele pode ser visto como a força propulsora e dinamizadora das ações de enfermagem nas mais diferentes dimensões e variações históricas ${ }^{(4)}$.

Ao voltarmos às fontes do conhecimento da disciplina* encontramos duas enfermeiras que embasaram e começaram a difundir o conhecimento da enfermagem: Catherine McAuley, junto a outras enfermeiras Irlandesas, no começo do século XIX, fundou em Dublin o Institute of Our Lady Mercy, estabelecendo serviços de enfermagem domiciliar e hospitalar. Suas experiências e conhecimentos foram registrados e são apresentados por autores como um modelo conceitual ${ }^{(7)}$. Florence Nightingale é responsável por escritos datados de 1859, considerados as primeiras teorias ou modelos conceituais desenvolvidos. Expressam o significado do processo de cuidado, a conceitualização de ambiente, a advertência para que as enfermeiras não focassem sua ação somente na doença (mas no doente e em seu entorno) e a distinção entre o enfoque teórico da enfermagem e o da medicina ${ }^{(8-11)}$.

Posteriormente, o grande avanço acerca da teorização do cuidado de enfermagem ocorreu logo após o fim da II Guerra Mundial, quando foram lançadas novas teorias e surgiram novas disciplinas. Tal fato promoveu um efeito imprevisto, mas salutar, na metodologia das ciências não físicas, subvertendo o modo tradicional de pesquisa nesses domínios e ressaltando o valor de teorias ${ }^{(12)}$. Assim, a partir da década de 1950, um grande número de enfermeiras teóricas desenvolveu modelos conceituais de enfermagem, oferecendo suporte para o desenvolvimento das teorias de enfermagem e do seu conhecimento ${ }^{(13-14)}$.
Essas mudanças também foram decorrentes das transformações na educação de enfermagem, a qual passou a fazer parte de estudos universitários, distanciando-se de uma formação vinculada apenas aos hospitais escola. Determinou-se, assim, um novo status para a disciplina e a profissão, promovendo novos modos de ensinar-aprender, além de contar com alunos provenientes de outras condições sociais, o que passou a determinar um novo perfil de egressos. A formação nesse contexto, anteriormente realizada por médicos, passou a ser exercida majoritariamente por enfermeiras. As idéias e conceitualizações da enfermagem passaram a receber atenção, e a formação passou a ser dirigida ao cuidado de enfermagem e ao paciente ${ }^{(8)}$.

No Brasil, na década de 1950 e início dos anos 1960, a necessidade de aperfeiçoamento da prática e a busca por novos conhecimentos exerceram grande ênfase na formação de recursos humanos de nível superior em instituições de ensino em países com sistema educativo mais desenvolvido, financiados por instituições de fomentos, como: Conselho Nacional de Desenvolvimento Científico e Tecnológico (CNPq), Coordenação de Aperfeiçoamento de Pessoal de Nível Superior (CAPES), Fundo do Desenvolvimento Técnico e Científico (FUNTEC), Fundação de Amparo à Pesquisa do Estado de São Paulo (FAPESP) e instituições internacionais, como a Fundação Rockfeller, a Ford e outras afins ${ }^{(15)}$. Esse processo de formação promoveu um avanço significativo da educação em enfermagem, já que os profissionais retornavam ao Brasil com conhecimento diferenciado e idéias inovadoras.

No final dos anos 1960, começam a se destacar as primeiras tentativas de sistematização das técnicas de enfermagem, seguidas pela preocupação em organizar princípios científicos que norteassem a prática profissional(16). A partir disso, houve a necessidade de aumento de qualificação dos profissionais e do Sistema Nacional de Pós-Graduação (SNPG). Assim, o Conselho Federal de Educação, através do Parecer Sucupira ${ }^{\circ}$ 977/65, aprova a Lei 5.540, que institui a Reforma Universitária Brasileira. Com isso, impulsionou-se a implantação de cursos de pós-graduação strictu-sensu (mestrado e doutorado) na área de enfermagem com vistas a qualificar docentes para atender as demandas do ensino superior e ampliar sua formação ${ }^{(17-18)}$.

Mas é na década de 1970, principalmente pelas contribuições das enfermeiras norte-americanas na área da educação e da administração, que se verifica uma importante fase de desenvolvimento da Enfermagem na América Latina, principalmente no Brasil, no Chile e na Colômbia ${ }^{(9,19)}$. A partir daí, a Enfermagem no Brasil começa a construir seu próprio corpo de conhecimento, começa a desenvolver pesquisas, a escrever teorias, modelos conceituais, metodologias e a aplicá-las tanto na academia como na prática. Na década de 1970 houve a formação de um maior número de mestres e, na de 1980, de doutores, consolidando a visibilidade da disciplina, com atividades de pesquisa e fortalecimento e ampliação do sistema nacional de pós-graduação em Enfermagem ${ }^{(18,20)}$.

Articulada ao desenvolvimento proveniente de estudos mais críticos publicados no Brasil a partir da década de 1980,

* Disciplina neste contexto significa uma ciência, enquanto objeto de aprendizado ou de ensino(6). 
ocorre a ampliação da produção científica na área de Enfermagem, tanto do ponto de vista de dissertações e teses, como de artigos publicados em periódicos indexados e livros. Esses fatos contribuem para a consolidação, no cenário educacional nacional e internacional, na área da saúde e da Enfermagem, da formação de recursos humanos qualificados e do desenvolvimento científico tecnológico nacional ${ }^{(21)}$.

A evolução do conhecimento de Enfermagem ocorreu historicamente e se consolidou pela elaboração e o aprimoramento de conhecimentos sobre o cuidado que a mesma presta ao ser humano. O conhecimento de Enfermagem foi construído por meio de conhecimento empírico e científico, e teve como fatores determinantes para a sua elaboração as tradições ou culturas, os empréstimos de teorias de outras disciplinas, o ensaio e o erro, as experiências pessoais, as influências de outros pesquisadores, a intuição, o raciocínio lógico e a pesquisa científica ${ }^{(22)}$.

A partir de toda essa construção do corpo de conhecimento da Enfermagem, foram elaboradas estruturas científicas que a embasam e que, dependendo do grau de abstração, recebem diferentes denominações. Assim, segundo a proposta de Fawcett (2005), as estruturas que compõem o conhecimento dessa disciplina foram desenvolvidas e classificadas em ordem decrescente de abstração: metaparadigma, filosofia, modelos conceituais, teorias e indicadores empíricos ${ }^{(11,23-24)}$. Tais estruturas são a base conceitual e filosófica da Enfermagem, e ainda possuem o objetivo de guiá-la dentro de bases metodológicas adequadas para a elaboração de seu conhecimento. Nessa proposição de estruturas do conhecimento não está contemplada a estrutura "modelo de cuidado de enfermagem", que aparece com maior ênfase no Brasil, em meados da década de 90 do século XX, em diferentes propostas de estudos ${ }^{(25-29)}$.

O modelo de cuidado é uma estrutura do conhecimento da Enfermagem, assim denominada por propor um desenho da realidade através da conceitualização e da proposição de seu conhecimento. Inclui também a proposição de um processo de cuidar, que é representado, geralmente, por modelos esquemáticos utilizando caixas de textos, figuras geométricas e outros símbolos ${ }^{(30)}$. Os modelos de cuidado de Enfermagem possuem as mesmas propriedades e demandam as mesmas necessidades para a sua elaboração que as demais estruturas do conhecimento dessa disciplina ${ }^{(31)}$.

Essa estrutura tem sido utilizada com diferentes terminologias e vem sofrendo transformações e aprimoramentos, podendo ser uma das razões para que não esteja consolidada e amplamente utilizada pela Enfermagem ${ }^{(31)}$.

Se pensarmos no grau de abstração que caracteriza um modelo de cuidado de Enfermagem, em consonância com a classificação das estruturas de conhecimento citada, ele estaria abaixo das teorias e acima dos indicadores empíricos, porque tal modelo é uma estrutura formada por um marco referencial (ou um marco teórico) e uma metodologia. Ademais, possui um nível de abstração inferior ao da teoria, pois pode ser derivado de uma ou mais teorias, além de apresentar uma possibilidade de planejamento e sistematização do cuidado de Enfermagem ${ }^{(31)}$. Sua elaboração pode ser realizada por meio de diferentes processos, mas todos seguem a lógica do raciocínio, seja indutivo, dedutivo ou indutivo-dedutivo. A escolha do método lógico está diretamente relacionada à concepção de cuidado e ao referencial teórico do enfermeiro. Nesse sentido, por acreditar que um modelo de cuidado deve contemplar o pragmatismo com o cientificismo e considerar o ser humano de modo holístico, a lógica indutiva-dedutiva pode ser o melhor caminho para a elaboração de um modelo, já que sua proposição se alicerça na realidade concreta dos sujeitos envolvidos: enfermeiro e pessoas que precisam de cuidados.

\section{PESQUISA CONVERGENTE ASSISTENCIAL}

Os modelos de cuidado de Enfermagem exigem para a sua elaboração um processo com coerência lógica, rigor científico e metodologia adequada, ou seja, um processo de pesquisa. E a pesquisa qualitativa, dentre elas a PCA, contribui na proposição de modelos de cuidado por propor, na elaboração de seu processo de construção, a lógica indutiva-dedutiva e uma relação direta com a prática assistencial.

Além disso, a PCA propõe a proximidade e o afastamento diante do saber-fazer assistencial, pois nos induz a uma metodologia em que há uma relação direta entre a academia e a prática. Devido a esse movimento, em alguns momentos há maior vínculo com o cuidado, e em outros, com a pesquisa. E é durante esse processo que ocorre a construção, ou o aprimoramento de um conhecimento, ou de uma proposta de cuidado ${ }^{(32)}$.

A PCA, proposta por Paim e Trentini e publicada pela primeira vez em 1999, tem a intencionalidade de unir métodos de pesquisa e métodos de prática assistencial. Originou-se da experiência das autoras na orientação de pesquisas de mestrado, nas quais buscavam uma articulação explícita entre a pesquisa e o cuidado ${ }^{(33-34)}$. Desde sua proposição, a PCA tem sido utilizada em diferentes cenários e com diferentes sujeitos, mas sempre vinculada diretamente ao contexto das práticas profissionais. Essa proposta metodológica vem sendo usada de modo crescente por várias enfermeiras nos seus projetos, sendo que a Região Sul do Brasil possui o maior número de publicações de estudos que utilizaram a $\mathrm{PCA}^{(32,35)}$.

Os princípios da PCA são: manter, durante seu processo, uma estreita relação com a prática assistencial, com o propósito de encontrar alternativas para solucionar ou minimizar problemas, realizar mudanças e/ou introduzir inovações no contexto da prática em que ocorre a investigação; o tema da pesquisa deve emergir das necessidades da prática reconhecidas pelos profissionais e/ou pelos usuários do campo da pesquisa; o pesquisador assume compromisso com a construção de um conhecimento novo para a renovação das práticas assistenciais no contexto estudado; a PCA deve ser desenvolvida no mesmo espaço físico e temporal da prática; os pesquisadores devem estar dispostos a se inserirem nas ações das práticas de saúde no contexto da pesquisa durante seu processo; a PCA permite a incorporação das ações de prática assistencial e/ou outras práticas relacionadas à saúde no processo de pesquisa e vice-versa; a pesquisa e a prática possuem identidades próprias que precisam ser consideradas durante o processo de investigação; aceita a utilização de vários e diferentes métodos e técnicas de coleta e análise dos dados $^{(34-35)}$. 
Portanto, a implementação de uma PCA requer que os pesquisadores desenvolvam simultaneamente pesquisa e cuidado com a intenção de provocar mudanças na assistência ${ }^{(32)}$. Desse modo, a PCA pode ser utilizada como caminho metodológico para a elaboração de modelos de cuidado com lógica indutiva-dedutiva, já que o enfermeiro, ao utilizar a PCA, precisa se colocar em compromisso com a construção de um novo conhecimento, de novos modos de cuidado e de novas tecnologias, promovendo a renovação ou inovação da prática assistencial.

A implementação da PCA ocorre por meio das seguintes fases: a concepção, a instrumentação, a perscrutação, a análise e a interpretação ${ }^{(33-34)}$. A fase de concepção representa a escoIha do tema, o direcionamento da questão guia, o estabelecimento dos objetivos da pesquisa, a revisão de literatura sobre o tema escolhido, a elaboração de conceitos e pressupostos, ou seja, é o marco referencial ou teórico.

A fase de instrumentação consiste na elaboração dos procedimentos metodológicos. Nessa etapa incluem-se a escolha do espaço da pesquisa, a escolha dos participantes e a escoIha da técnica para obtenção e análise das informações. A fase de perscrutação inclui a coleta e o registro dos dados, que se destinam a obter informações com dupla intencionalidade: produzir construções científicas nas atividades de pesquisa e favorecer o aperfeiçoamento do cuidado prestado pela Enfermagem. A fase de análise da PCA é dividida em duas etapas a análise e a interpretação.

Na etapa análise, temos o processo de apreensão, na etapa interpretação, encontramos os processos de síntese, teorização e recontextualização. O processo de síntese, incluído na etapa interpretação, consiste em examinar subjetivamente os dados e realizar associações e variações das informações, encontradas no processo de apreensão. O processo de teorização consiste em descobrir os valores contidos nas informações levantadas durante o processo de síntese. A teorização se faz pela relação da fundamentação teórico-filosófica utilizada no estudo de maneira a proceder à associação com os dados. Assim, formulam-se novos conceitos, definições e inter-relações, constituindo as conclusões do estudo. O processo de recontextualização consiste na possibilidade de dar significado a determinados achados ou descobertas e procurar contextualizá-los em situações similares, ou seja, é a socialização de resultados singulares ${ }^{(33-34)}$.

\section{PROPONDO MODELOS DE CUIDADO: A CONTRIBUI- ÇÃO DA PESQUISA CONVERGENTE ASSISTENCIAL}

A necessidade da elaboração de um modelo de cuidado pode advir de problemas encontrados durante a realização do cuidado na prática ou na teoria, ou seja, durante pesquisas ou durante estudos. Essa inquietação é decorrente de diferentes problemas, como a falta de organização na assistência, a realização de um cuidado não embasado cientificamente, a necessidade de garantir a segurança do paciente, a falta de um processo de cuidado sistematizado, a falta de recursos tecnológicos, a necessidade da elaboração de novas tecnologias de cuidado, entre outros.

A partir disso, o enfermeiro identifica o problema e escolhe o tipo e a metodologia de pesquisa adequada para embasar sua construção teórica de elaboração de um modelo de cuidado.

Ao pensar em construir o modelo utilizando a lógica indutivo-dedutiva, a PCA torna-se uma escolha adequada para tal processo, pois indica que o modelo será construído a partir da realidade, relacionando pragmatismo e cientificismo durante todo seu processo de elaboração.

Para o preparo de um modelo de cuidado utilizando essa metodologia, o enfermeiro precisa manter estreita relação com a prática assistencial, verificando por que, como, onde, com quem, quanto e como a situação problema se apresenta. A partir daí, busca aprofundar seu conhecimento, utilizando vivências, experiências profissionais e sensibilidade, realizando um processo de construção mental a partir de um conhecimento específico ao mesmo tempo que engloba diversas dimensões, embasando a elaboração de um conhecimento e de ações que solidificarão o empirismo em um cuidado científico, específico e sistematizado. Assim, a PCA, nas fases de concepção e de instrumentação, nos proporciona a elaboração inicial da construção de um modelo. Esse é o momento em que o enfermeiro encontra o problema que o faz refletir e tomar determinadas decisões teóricas e metodológicas.

A elaboração de um marco referencial (ou marco teórico) que irá orientar a prática assistencial identifica o eixo teórico e filosófico do modelo de cuidado. Esta construção teórica proporciona ao profissional a evidência que ele necessita para embasar, direcionar e clarificar suas ações, apontando e justificando a seleção de determinado problema de estudo ${ }^{(10)}$. $\mathrm{O}$ marco referencial visa aprofundar formas de orientar as ações de Enfermagem por meio dos conceitos, formalizando, assim, uma construção mental logicamente organizada.

A construção de um marco referencial ou um marco teórico se dá pela abrangência do tema proposto. $\mathrm{O}$ enfermeiro verifica se uma ou mais teorias abrangem todas as dimensões que a proposta do estudo necessita ou se é necessário buscar suporte no conhecimento de outras disciplinas afins. É nesse momento que a base teórico-filosófica do modelo começa a ser delineada.

Durante a elaboração do marco referencial (ou teórico), o profissional elege os conceitos centrais e importantes para a compreensão do modelo, dentre eles, o cuidado de Enfermagem. Nesse sentido, é necessário explicitar qual é a compreensão do enfermeiro sobre o fenômeno em foco (violência, cirurgia, nascimento, educação, entre outros), do sujeito/ cliente (criança, mulher, idosos, adolescentes, alunos, entre outros), do ambiente, da Enfermagem, da saúde, entre outros. Nesse momento o enfermeiro explicita os pressupostos de seu estudo.

Pressupostos são declarações de crença do enfermeiro relativas ao fenômeno em estudo, ou seja, uma explicitação sobre a partir de que ponto de vista e de que concepções serão estabelecidas as suas ações de cuidado. Isso define em que paradigma estão assentadas as ações de cuidado, sua natureza, sua abrangência e as disciplinas envolvidas. É necessário destacar que o marco referencial (ou teórico) vai sendo aperfeiçoado no decorrer da elaboração do modelo. O marco referencial (ou teórico) serve também como base para a elaboração 
da metodologia, assumindo grande visibilidade, pois orienta a decisão/escolha das ações/intervenções a serem realizadas, requerendo que o enfermeiro possua uma base teórica que a guie nessa caminhada.

A fase de instrumentação corresponde ao momento de definição dos modos de intervenção/ações de cuidado a serem implementadas, ou seja, ao momento em que a metodologia do modelo de cuidado é desenhada.

As fases de perscrutação, de análise e de interpretação da PCA, ao oferecerem novos subsídios sobre a aplicabilidade do referencial na prática assistencial, conduzem ao processo de revisão do que foi proposto inicialmente.

Os modelos de cuidado requerem a proposição de uma representação gráfica que favoreça seu entendimento e sua aplicabilidade no cotidiano da prática profissional, explicitando o relacionamento entre os conceitos. Essa construção pode ser desenvolvida inicialmente na fase de instrumentalização, e sua validação pode ser iniciada durante a fase de perscrutação da PCA, que permite o confronto da realidade com a proposta do modelo que está sendo construído. Assim, ao aplicar o modelo de cuidado na prática (fase de perscrutação da PCA), o enfermeiro verifica sua validade e o quanto está ajustado à realidade da qual emergiu, indicando possíveis adequações para torná-lo um modelo de fácil compreensão e implementação, que favoreça o cuidado profissional exercido pelo enfermeiro. Para isso, também deve incluir as informações essenciais que garantam a excelência e a eficiência do cuidado prestado.

Simultaneamente à implementação de ações/intervenções de cuidado na fase de perscrutação, ocorrem as fases de análise e de interpretação. Na fase de análise dá-se o processo de apreensão, e os dados são organizados de forma a permitir que o enfermeiro analise a validade de suas ações, e eleja, se for preciso, outras ações necessárias e não contempladas a priori. É um modo de monitoramento das ações de cuidado desenvolvidas e de verificação de sua eficácia frente aos objetivos propostos.

Assim, após a organização dos dados inicia-se a fase de interpretação, de extrema importância para a realização dos ajustes na metodologia e no marco referencial (ou teórico) propostos para o modelo de cuidado, sendo que a metodologia pode ser ajustada, e os métodos, revistos. Essa fase finaliza o processo de elaboração do modelo de cuidado de enfermagem.

Devemos destacar que esse processo necessita de tempo para sua elaboração e aperfeiçoamento, que poderá levar à construção de um novo conhecimento ou uma nova tecnologia. Necessita de tempo também para sua aplicação e para a assimilação do novo processo estabelecido. Além disso, o rigor científico e a ética devem permear a construção de todo esse processo.

Os modelos de cuidado podem ser elaborados para atender demandas dos diferentes cenários do cuidado da Enfermagem, com diferentes clientes em diferentes condições de saúde. A elaboração de modelos de cuidado nos reporta a uma imensidão de possibilidades, quais sejam, elaborar ou aperfeiçoar um cuidado ou processo de cuidado, criar novas tecnologias de cuidado, aplicar o cuidado proposto, receber uma resposta do entorno em que foi aplicado, ser elaborado por enfermeiros assistenciais ou pesquisadores, ser aplicado por alunos de Enfermagem, entre outros. A PCA nos auxilia nesse processo, pois considera a objetividade, a subjetividade, a praticidade, a adequação e a segurança, que devem ser inerentes no cuidado prestado. Esse processo de elaboração é dinâmico, assim como a PCA, com isso as diferentes fases não ocorrem necessariamente em uma sequência rígida, mas podem ocorrer concomitantemente.

\section{REFLETINDO ACERCA DO MODELO DE CUIDADO COMO UM ALICERCE PARA O CUIDADO PROFISSIO- NAL DE ENFERMAGEM}

A construção de modelos de cuidado de Enfermagem perpassa por diferentes dimensões e necessita estar voltada para a prática e a teorização do cuidado de forma a contribuir para a sua sistematização. Melhor dizendo, a produção do conhecimento deve resultar do processo de criação conjunta do ensino, da prática assistencial e da gestão, com a participação ativa da clientela e considerando as crenças e os valores dos participantes. Esse processo deve ser permeado por avaliações críticas acerca da natureza dos pressupostos filosóficos que norteiam a prática ${ }^{(36)}$.

A consolidação do corpo de conhecimento da Enfermagem requer um trabalho reflexivo do enfermeiro, pois pensar no cuidado de Enfermagem como o lugar de cuidado às pessoas é pensar complexamente na ambiguidade das relações entre o enfermeiro e o sujeito. A Enfermagem tem um compromisso consigo mesma (de construção de seu corpo de conhecimentos) e com a humanidade, no sentido de "reconstruir" sua prática valorizando a fala, as respostas e as experiências humanas nos diferentes ciclos da vida ${ }^{(37)}$.

Por isso, a proposição de Modelos de Cuidado elaborados a partir de processos indutivo-dedutivos representa um importante alicerce para o cuidado profissional de Enfermagem, podendo contribuir para a consolidação do seu corpo de conhecimento $\mathrm{e}$, em especial, à sintonia do cuidado às necessidades concretas dos sujeitos cuidados. Isso porque todas as ações do enfermeiro na produção de assistência devem se efetivar por meio do cuidado, da educação, da informação, da comunicação e do gerenciamento, tendo a finalidade de atender necessidades da população relacionadas à manutenção da saúde como condição de sua natureza de ser vivo ${ }^{(38)}$.

Portanto, a importância da incorporação de modelos de cuidado (envolvendo sua elaboração e implementação) na estrutura do conhecimento de enfermagem se dá pela possibilidade de contribuir para uma prática reflexiva que permite ao enfermeiro propor modos de cuidado aderentes à realidade concreta de sua prática, além de favorecer a avaliação dos resultados de sua ação. $O$ processo de elaboração de modelos alia o cuidado, o ensino e a pesquisa, desvelando a inerência dessa articulação. Os modelos sistematizam o cuidado e favorecem a unidualidade entre a academia e a prática do cuidado, além de contribuírem para que as ações de Enfermagem sejam pautadas na cientificidade e na segurança do cuidado prestado. 


\section{REFERÊNCIAS}

1. Neves EP. As Dimensões do Cuidar em Enfermagem: concepções teórico-filosóficas. Esc Anna Nery Rev Enferm 2002;6(1):79-92.

2. Rocha PK, Prado ML, Wal ML, Carraro TE. Cuidado e Tecnologia: aproximações através do modelo de cuidado. Rev Bras Enferm 2008;61(1):113-6.

3. Colliére MF. Promover a vida - da prática das mulheres de virtude aos cuidados de enfermagem. Tradução de Maria Leonor Braga Abecasis. Damaia: Printipo Indústrias Gráficas Ltda; 1989. p. 385.

4. Backes, DS, Sousa FGM, Mello ALSF, Erdmann AL, Nascimento KC, Lessmann JC. Concepções de Cuidado: uma análise das teses apresentadas para um programa de pós-graduação em enfermagem. Texto \& Contexto Enferma 2006;15(n. ${ }^{\circ}$ esp.):71-8.

5. Aimar A, Videla N, Torre M. Tendencias y Perspectivas de la Ciencia Enfermera. Enferm Glob [periódico na internet]. 2006 [acesso em 17 set 2009];3(9) Disponível em: < http://revistas.um.es/eglobal/article/view/385/346>

6. Abbagnano N. Dicionário de Filosofia. 4. ed. São Paulo: Martins Fontes; 2000. p.1010.

7. Meehan TC. Careful Nursing: a model for contemporary nursing practice. J Adv Nurs 2003;44(1):99-107.

8. Riehl JP, Roy C. History of Nursing Models. Conceptual Models Nursing Practice. New York: Appleton-CenturyCrofts; 1974.

9. Villalobos MMD. Enfermería: desarrollo teórico e investigativo. Colombia: Universidad Nacional de Colombia; 1998. p. 172.

10. Carraro TE. Sobre teorias e marco conceitual: sua influência na metodologia da assistência. In: Carraro TE, organizadores. Metodologias para a assistência de enfermagem: teorização, modelos e subsídios para a prática. Goiânia: $A B ; 2001$. p. 29-38.

11. Fawcett J. Knowledge Contemporary Nursing Knowledge: analysis and evolution of nursing models and theories. 2. ed. Philadelphia: F. A. Davis Company; 2005. p. 623.

12. Bunge M. Teoria e realidade. São Paulo: Perspectiva; 1974. p. 243.

13. Alligood MR. Philosophies, Models, and Theories: Critical Thinking Structures. In: Alligood MR, Tomey AM, organizadores. Nursing Theory - Utilization \& Application. 3. ed. Missouri: Mosby Elsevier; 2006.

14. Cometto MC, Piovan M, Gómez P. Aportes de los Coloquios Panamericanos a la Investigación en Enfermería - Período 2000-2006. Texto \& Contexto Enferm 2008; 17(4):720-6.

15. Padilha MICS, Borenstein MS, Maia AR, Guedes JAD, Lessmann JC, Machado CA. Uma História de Sucesso: 30 Anos da Pós-Graduação em Enfermagem da UFSC. Texto \& Contexto Enferm 2006; 15 (n. ${ }^{\circ}$ esp.):20-30.

16. Paim L, Prado ML, Silva DMGV, Carraro TE, Mazza VA. Conceitos e visões teóricas. Florianópolis: REPENSUL:
ESPENSUL, 1998. p. 140.

17. Rodrigues RAP, Erdmann AL, Fernandes JD, Araújo TL. Pós-graduação em enfermagem no Brasil e no Nordeste. Revista Gaúch Enferm 2007;28(1):70-8.

18. Rodrigues RAP, Erdmann AL, Silva IA, Fernandes JD, Araújo TL, Vianna LAC, Santos RS, Lopes MJM. Educação do Doutorado em Enfermagem no Brasil. Rev Latino-Am Enferm 2008;16(4):665-71.

19. Villalobos MMD. Teorías de rango medio: ¿ Por qué para el cuidado? Importancia y utilidad para la práctica de enfermería. In: El arte y la ciencia del cuidado. Colombia: Universidad Nacional de Colombia; 2002. p. 32.

20. Erdmann AL. Desafios da Enfermagem na CAPES: produtos altamente qualificados, Rev Esc Enferm USP 2008;42(2):216-7.

21. Padilha MICS, Kletemberg DF, Gregório VRP, Borges LM, Borenstein MS. A Produção da Pesquisa Histórica Vinculada aos Programas de Pós-Graduação no Brasil, 1972 a 2004. Texto \& Contexto Enferm 2007;16(4):671-9.

22. Burns N, Grove SK. Investigación en Enfermería. Espanha: Editora Elsevier; 2005.

23. Polit DF, Beck CT. Nursing Research: generating and assessing evidence for nursing practice. Filaphelfia: Wolters Kluwer/Lippicott Willians \& Wilkins; 2008. p. 350.

24. Meleis Al. Theoretical Nursing Development and Progress. 3. ed. Philadelphia: Lippincott; 1997.

25. Erdmann AL. Diagnóstico de problemas num sistema de enfermagem de hospital de ensino: proposta de um modelo. Rio de Janeiro: UERJ; 1987.

26. Zanchetta MS. O processo de avaliação de um modelo assistencial em cancerologia: modelo assistencial. Rio de Janeiro: UERJ; 1990.

27. Carraro TE. Resgatando Florence Nightingale: a trajetória da Enfermagem junto ao ser humano e sua família na prevenção de infecções. Florianópolis. Dissertação [Mestrado em Enfermagem]- Universidade Federal de Santa Catarina. Programa de Pós-Graduação em Enfermagem; 1994.

28. Carraro TE, Wall ML. Um modelo de cuidado de enfermagem aplicado a grupos de mulheres-mães. Av Enferm 2005;23(1):90-9.

29. Teixeira MA, Nitschke RG. Modelo de Cuidar em Enfermagem junto as Mulheres-avós e sua Família no Cotidiano do Processo de Amamentação. Texto \& Contexto Enferm 2008;17(1):183-91.

30. Rocha PK. Construção e Validação de um Instrumento para Avaliação de Modelos de Cuidado de Enfermagem. Florianópolis. Tese [Doutorado em Enfermagem]- Universidade Federal de Santa Catarina. Programa de Pós-Graduação em Enfermagem; 2008.

31. Rocha PK, Prado ML. Modelo de Cuidado ¿Qué es y como elaborarlo? Index Enferme 2008;17(2):128-32.

32. Paim L, Mercedes T, Madureira VSF, Stamm M. Pesquisa Convergente-Assistencial e sua Aplicação em Cenários da 
Enfermagem. Cogitare Enfermagem. 2008;13(3):380-6.

33. Trentini M, Paim L. Pesquisa em enfermagem - uma modalidade convergente-assistencial. Florianópolis: UFSC; 1999. p. 162.

34. Trentini M, Paim L. Pesquisa convergente assistencial: um desenho que une o fazer e o pensar na prática assistencial em saúde-enfermagem. 2. ed. Florianópolis: Insular; 2004. p. 143.

35. Trentini M, Beltrame V. A Pesquisa Convergente-Assistencial (PCA) levada ao real campo de ação da Enfermagem. Cogitare Enferm 2006;11(2):156-60.
36. Silva AL, Arruda EN. Referenciais com Base em Diferentes Paradigmas: problema ou solução para a prática de Enfermagem? Texto \& Contexto Enferm 1993;3(1):82-92.

37. Azevedo RCS, Ramos FRS. Modos de conhecer e intervir: a constituição do corpo no cuidado de enfermagem no hospital. Texto \& Contexto Enferm 2006;15(n. ${ }^{\circ}$ esp.):55-63.

38. Cunha RR, Pereira LS, Gonçalves ASR, Santos EKA, Radunz V, Heidemann ITSB. Promoção da saúde no contexto paroara: possibilidade de cuidado de enfermagem. Texto \& Contexto Enferm 2009;18(1): 170-6. 